\title{
Continuity and Monotonicity of the MPC Value Function with respect to Sampling Time and Prediction Horizon *
}

\author{
Vincent Bachtiar $^{\mathrm{a}}$, Eric C. Kerrigan ${ }^{\mathrm{b}}$, William H. Moase ${ }^{\mathrm{a}}$, Chris Manzie ${ }^{\mathrm{a}}$ \\ ${ }^{a}$ Dept of Mechanical Engineering, The University of Melbourne, VIC 3010, Australia \\ ${ }^{\mathrm{b}}$ Dept of Electrical and Electronic Engineering and Dept of Aeronautics, Imperial College London, London SW7 2AZ, U.K.
}

\begin{abstract}
The digital implementation of model predictive control (MPC) is fundamentally governed by two design parameters; sampling time and prediction horizon. Knowledge of the properties of the value function with respect to the parameters can be used for developing optimisation tools to find optimal system designs. In particular, these properties are continuity and monotonicity. This paper presents analytical results to reveal the smoothness properties of the MPC value function in open- and closed-loop for constrained linear systems. Continuity of the value function and its differentiability for a given number of prediction steps are proven mathematically and confirmed with numerical results. Non-monotonicity is shown from the ensuing numerical investigation. It is shown that increasing sampling rate and/or prediction horizon does not always lead to an improved closedloop performance, particularly at faster sampling rates.
\end{abstract}

Key words: Control system design, Optimal control, Optimisation

\section{Introduction}

When designing a model predictive controller (MPC), it is important to consider the influence of various design choices on both the performance and computational complexity of the controller. These design choices could include attributes of the optimal control problem (OCP) associated with the MPC such as sampling time, prediction horizon and model order, as well as attributes of the numerical routine used to solve the OCP. Since sampling and horizon times are quantities that must be chosen in any implementation of MPC, this discussion will focus on the influence of these parameters.

A common practice aimed at improving MPC performance is to decrease sampling and/or increase horizon times, typically whilst meeting computational requirements for real-time controller implementation. There is no guarantee that relying upon this 'conventional wisdom' will improve performance. It has been previously established for linear (and nonlinear [7]) quadratic regu-

\footnotetext{
* This paper was not presented at any IFAC meeting. The corresponding author is V. Bachtiar.

Email addresses: bachtiarv@unimelb.edu.au (Vincent Bachtiar), e.kerrigan@imperial.ac.uk (Eric C. Kerrigan), moasew@unimelb.edu.au (William H. Moase), manziec@unimelb.edu.au (Chris Manzie).
}

lators (LQR) that decreasing sample time or increasing prediction horizon can degrade closed-loop performance $[7,8]$. For MPC, where constraints and finite horizons complicate matters, a preliminary analysis not considering state constraints and a terminal cost [1] have echoed the LQR results. A formalised and rigorous analysis of closed-loop MPC performance has yet to be addressed.

This paper considers the continuity, differentiability and monotonicity of control performance in constrained linear systems, measured by an open- or closed-loop value function, with respect to the sample and horizon times. This follows the spirit of related research exploring the open-loop value function sensitivity, and showing that it is piecewise quadratic with respect to the current state of the plant $[3,4]$. In this paper, an MPC is formulated initially in continuous-time with a given prediction horizon and a zero-order-held input, following [5]. The discretetime equivalence is then formulated, with a residual sampling interval to account for when the prediction horizon is not an integer multiple of the sampling time.

Knowledge of the smoothness properties of the MPC performance with respect to the design parameters is useful to develop systematic methods such as optimisation tools, in place of the conventional wisdom, to select sampling and horizon times that achieve the best control performance and alleviate online computational 
cost. For example, a gradient-based method might be ineffective for a discontinuous optimisation surface and a global optimiser is useful if the surface is non-monotonic.

Perturbation analysis is used below to prove continuity of the value function and its differentiability for a given number of prediction steps. Numerical results are presented to confirm the analytical results and demonstrate non-monotonicity, thereby providing examples where conventional wisdom may result in worse MPC designs.

Notational conventions and definitions: For $M \in \mathbb{R}^{n \times n}$, $v \in \mathbb{R}^{n \times m}$ and $s \in \mathbb{R}$ with appropriate $n$ and $m,\|v\|_{M}^{2}:=$ $v^{\top} M v$. Superscript + denotes the function value after its argument(s) is perturbed, e.g. $M^{+}:=M\left(s+\delta_{s}\right) \cdot \partial_{s} v:=$ $\left.\frac{\partial v}{\partial s}\right|_{s}$ is the derivative of a differentiable matrix $s \mapsto$ $v(s)$ evaluated at $s$. Big- $\mathcal{O}$ notation; $f(x)=\mathcal{O}(g(x)) \Leftrightarrow$ $\exists a \in \mathbb{R}_{>0}, x_{0} \in \mathbb{R}_{>0}:|f(x)| \leq a|g(x)| \forall x \in\left[0, x_{0}\right)$. Let $\mathcal{O}\left(g_{1}(\cdot), \ldots, g_{n}(\cdot)\right)=\sum_{i=1}^{n} \mathcal{O}\left(g_{i}(\cdot)\right) . \lambda_{i}(M)$ is the $i^{\text {th }}$ eigenvalue of the matrix $M$. $\Im(\cdot)$ denotes the imaginary part of its argument.

Definition 1 (Continuity [11]) $f(\cdot): \mathbb{R}^{n} \rightarrow \mathbb{R}$ is continuous at $s \in \mathbb{R}^{n}$ if $\lim _{x \rightarrow s} f(x)=f(s)$.

Definition 2 (Differentiability [11]) $f(\cdot): \mathbb{R}^{n} \rightarrow \mathbb{R}$ is differentiable at $s \in \mathbb{R}^{n}$ if it can be linearly approximated in the neighbourhood of the point, i.e.

$$
f(s+\delta)=f(s)+\Delta(s) \cdot \delta+L(s, \delta) \cdot \delta,
$$

where the gradient $\Delta(s) \in \mathbb{R}^{n}$ is independent of $\delta$, and $\lim _{\delta \rightarrow 0} L(s, \delta)=0, \forall s \in \mathbb{R}^{n}$ constitutes higher order terms. Differentiability at point s implies continuity at $s$.

\section{Problem Formulation}

\subsection{Definition of $O C P$}

Consider a linear time-invariant dynamic plant model $\dot{\mathrm{x}}(t)=A \mathrm{x}(t)+B \mathrm{u}(t)$ with states $\mathrm{x}(t) \in \mathbb{R}^{n_{x}}$ and inputs $\mathrm{u}(t) \in \mathbb{R}^{n_{u}}$. Discretisation is required for the purpose of digital control; the plant is controlled in a sampled-data fashion at sampling instants $t_{i}, i \in \mathbb{N}_{\geq 0}$, with sampling interval $h$. A sampling-to-actuation delay of zero is assumed. The control input is restricted to a zero-orderhold $(\mathrm{ZOH}) ; \mathrm{u}(t)=\mathrm{u}_{i}, \forall t \in[i h, i h+h), i \in \mathbb{N}_{\geq 0}$.

In the context of optimal regulation, the control command can be obtained by solving a finite-horizon, optimal control problem (OCP) at each sampling instant $i$;

$$
\begin{aligned}
& \min _{(u, x)} \int_{0}^{T}\left(\|x(\tau)\|_{Q}^{2}+\|u(\tau)\|_{R}^{2}\right) d \tau+\|x(T)\|_{Q_{f}}^{2} \\
& \text { s.t. } x(0)=\mathrm{x}\left(t_{i}\right) \\
& \dot{x}(\tau)=A x(\tau)+B u(\tau) \quad \forall \tau \in[0, T] \\
& x(\tau) \in[\check{x}, \hat{x}], u(\tau) \in[\check{u}, \hat{u}] \forall \tau \in[0, T) \\
& u(\tau)=u(k h), \forall k \in \mathbb{N}_{\geq 0} \quad \forall \tau \in[k h, k h+h)
\end{aligned}
$$

Note the distinction between the real-time variable $x$ and predictive variable $x$ used. $h$ is the sampling interval and $T$ is the prediction horizon. Dependence on these is ubiquitous in subsequent derivations and, when obvious, not explicitly written to maintain notational succinctness.

The cost (1a) consists of the stage cost weighted by $Q \geq$ 0 and $R>0$, and terminal cost weighted by $Q_{f} \geq 0$ to penalise the state/input deviations from zero. The predictive model (1c) and constraints (1d) represent the dynamics and limitations of the plant. The $\mathrm{ZOH}$ control (1e) discretises the control command over the sampling steps $k \in\{0, \ldots, N\}$ with $N:=\lfloor T / h\rfloor \in \mathbb{N}_{>0}$. The 'residual sampling interval' is denoted as $h_{\mathrm{r}}:=T-N h$. Note that no terminal constraint is imposed and stability is guaranteed only by the use of a terminal cost.

The plant can be represented in discrete-time with $\mathrm{x}_{i+1}=\bar{A} \mathrm{x}_{i}+\bar{B} \mathrm{u}_{i}, \forall i \in \mathbb{N}_{\geq 0} \cdot \mathrm{x}_{i} \in \mathbb{R}^{n_{x}}$ and $\mathrm{u}_{i} \in \mathbb{R}^{n_{u}}$ are the states and inputs at sampling instant $i$. The state transition matrices are

$$
\bar{A}:=\Phi(h):=e^{A h}, \quad \bar{B}:=\Gamma(h):=\int_{0}^{h} e^{A(h-\tau)} d \tau B .
$$

$\bar{A}_{\mathrm{r}}:=\Phi\left(h_{\mathrm{r}}\right)$ and $\bar{B}_{\mathrm{r}}:=\Gamma\left(h_{\mathrm{r}}\right)$ for the residual sampling interval. The predicted states and input are presented in discrete-time with the vectors $\mathbf{x}:=\left[x_{0}^{\top} \cdots x_{N+1}^{\top}\right]^{\top}$ and $\mathbf{u}:=\left[u_{0}^{\top} \cdots u_{N}^{\top}\right]^{\top}$. The following representation of (1d); $x_{k} \in[\check{x}, \hat{x}], \forall k \in\{0, \ldots, N+1\}$, and $u_{k} \in[\check{u}, \hat{u}], \forall k \in$ $\{0, \ldots, N\}$ can be used. Note that this only guarantees state constraint satisfaction at sampling instances.

Consequently, the continuous-time OCP (1) is replaced by a quadratic program $(\mathrm{QP})$ in the form

$$
\begin{aligned}
& \min _{(\mathbf{u}, \mathbf{x})} \ell\left(\mathrm{x}_{i}, \mathbf{u}, \mathbf{x}\right)
\end{aligned}
$$

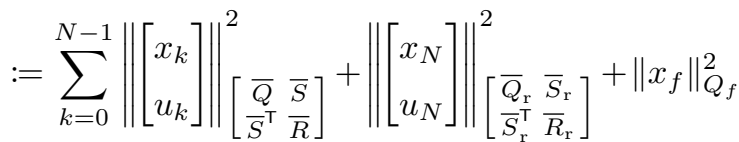

$$
\begin{aligned}
& \text { s.t. } x_{0}=\mathrm{x}_{i} \\
& x_{k+1}=\bar{A} x_{k}+\bar{B} u_{k} \quad \forall k \in\{0, \ldots, N-1\} \\
& x_{f}=\bar{A}_{\mathrm{r}} x_{N}+\bar{B}_{\mathrm{r}} u_{N} \\
& x_{k} \in[\check{x}, \hat{x}], u_{k} \in[\check{u}, \hat{u}] \quad \forall k \in\{0, \ldots, N\} \\
& x_{f} \in[\check{x}, \hat{x}]
\end{aligned}
$$

The open-loop value function and OCP solution are

$$
\begin{aligned}
J\left(h, T, \mathrm{x}_{i}\right) & :=\min _{(\mathbf{u}, \mathbf{x})} \ell\left(\mathrm{x}_{i}, \mathbf{u}, \mathbf{x}\right) \text { s.t. }(2 \mathrm{~b}-\mathrm{d}) \\
\left(\mathbf{u}^{*}\left(\mathrm{x}_{i}\right), \mathbf{x}^{*}\left(\mathrm{x}_{i}\right)\right) & :=\arg \min _{(\mathbf{u}, \mathbf{x})} \ell\left(\mathrm{x}_{i}, \mathbf{u}, \mathbf{x}\right) \text { s.t. }(2 \mathrm{~b}-\mathrm{d}) .
\end{aligned}
$$

Note the $(h, T)$ dependence of $\ell, \mathbf{x}^{*}$ and $\mathbf{u}^{*}$ is not written explicitly. The $2^{\text {nd }}$ term in (2a) is used to account for the cost integral (1a) in the interval $[0, T]$ when $T / h \notin \mathbb{N}_{>0}$. The $3^{\text {rd }}$ term is the more commonly known terminal cost, 
also used in (1a), that if appropriately chosen makes the cost function replicate an infinite-horizon cost to help provide stability guarantees [9].

The cost of the discrete-time problem (2) can be made equivalent to the continuous-time one (1) by setting [5]

$$
\bar{Q}:=\Xi_{Q}(h), \quad \bar{R}:=\Xi_{R}(h), \quad \bar{S}:=\Xi_{S}(h),
$$

and $\bar{Q}_{\mathrm{r}}:=\Xi_{Q}\left(h_{\mathrm{r}}\right), \bar{R}_{\mathrm{r}}:=\Xi_{R}\left(h_{\mathrm{r}}\right), \bar{S}_{\mathrm{r}}:=\Xi_{S}\left(h_{\mathrm{r}}\right)$ where

$$
\begin{aligned}
& \Xi_{Q}(h):=\int_{0}^{h}\|\Phi(\tau)\|_{Q}^{2} d \tau, \Xi_{S}(h):=\int_{0}^{h} \Phi(\tau)^{\top} Q \Gamma(\tau) d \tau, \\
& \Xi_{R}(h):=\int_{0}^{h}\|\Gamma(\tau)\|_{Q}^{2} d \tau+h R .
\end{aligned}
$$

The solution to the OCP (2) is applied in closed-loop in a receding horizon fashion with the associated control law

$$
\mathrm{u}_{i}=u_{0}^{*}\left(h, T, \mathrm{x}_{i}\right) .
$$

To define the 'closed-loop value function', first let

$$
\left.\ell_{0}\left(h, T, \mathrm{x}_{i}\right):=\left\|\left[\begin{array}{c}
\mathrm{x}_{i} \\
u_{0}^{*}\left(\cdot, \mathrm{x}_{i}\right)
\end{array}\right]\right\|^{2} \begin{array}{cc}
\bar{Q} & \bar{S} \\
\bar{S}^{\top} & \bar{R}
\end{array}\right] .
$$

The closed-loop value function is given analytically as

$$
\begin{gathered}
V\left(h, T, \mathrm{x}_{0}\right):=\sum_{i=0}^{\infty} \ell_{0}\left(h, T, \mathrm{x}_{i}\right) \\
\text { s.t. } \mathrm{x}_{i+1}=\bar{A} \mathrm{x}_{i}+\bar{B} u_{0}^{*}\left(h, T, \mathrm{x}_{i}\right) \quad \forall i \in \mathbb{N}_{\geq 0} .
\end{gathered}
$$

The closed-loop value function is an infinite series. Its convergence relies on the stability of the system as discussed later in Lemma 11. To numerically measure performance, a closed-loop simulation must be performed in finite time $t \in\left[0, T_{\text {sim }}:=N_{\text {sim }} h\right]$. The infinite series is truncated after $N_{\text {sim }} \in \mathbb{N}_{>0}$ steps.

The MPC value functions have now been formulated. Amongst their arguments, the design parameters $h$ and $T$ are the subjects of the investigation. Other design variables are kept constant.

\subsection{Solution to the $O C P$}

Both the open- and closed-loop value functions are functions of the solution $\mathbf{x}^{*}$ and $\mathbf{u}^{*}$. This can be classified by writing the OCP (2) in standard QP form. First let

$$
\mathbf{z}:=\mathbf{u}+\mathcal{R} \mathbf{x}+H^{-1} F^{\top} x_{i}
$$

where $\mathcal{R}:=\operatorname{diag}\left(\bar{R} \bar{S}^{\top}, \ldots, \bar{R} \bar{S}^{\top}, \bar{R}_{\mathrm{r}} \bar{S}_{\mathrm{r}}^{\top}\right), \mathbf{z}=:\left[z_{0}^{\top} \cdots z_{N}^{\top}\right]^{\top}$ and $\mathbf{z} \in \mathbb{R}^{N n_{u}} . H:=2\left(\widetilde{\mathbf{B}}^{\top} \widetilde{\mathbf{Q}} \widetilde{\mathbf{B}}+\mathbf{R}\right)>0$ is invertible as a consequence of the cost weight restrictions $Q \geq 0$, $R>0$ and $Q_{f} \geq 0$. Further, let the block matrices

$H$ be as above, $L:=2 \widetilde{\mathbf{A}}^{\top} \widetilde{\mathbf{Q}} \widetilde{\mathbf{A}}-F H^{-1} F^{\top}, F:=2 \widetilde{\mathbf{A}}^{\top} \widetilde{\mathbf{Q}} \widetilde{\mathbf{B}}$ with block transition matrices and block cost weights

$$
\begin{gathered}
\widetilde{\mathbf{A}}:=\left[\begin{array}{c}
\widetilde{\widetilde{A}} \\
\widetilde{A}_{\mathrm{r}}^{\widetilde{A}^{N}}
\end{array}\right], \quad \widetilde{\mathbf{B}}:=\left[\begin{array}{cccc}
\widetilde{A}_{\mathrm{r}} \widetilde{A}^{N-1} \bar{B} & \widetilde{A}_{\mathrm{r}} \widetilde{\widetilde{A}}^{N-2} \bar{B} & \ldots & \bar{B}_{\mathrm{r}}
\end{array}\right], \\
\underline{\widetilde{A}}:=\left[\begin{array}{c}
\widetilde{\widetilde{A}}^{0} \\
\widetilde{A}^{1} \\
\vdots \\
\widetilde{A}^{N}
\end{array}\right], \quad \underline{\widetilde{B}}:=\left[\begin{array}{cccc}
0 & \cdots & & \vdots \\
\widetilde{A} \bar{B} & \bar{B} & \\
\vdots & & \ddots & \vdots \\
\widetilde{A}^{N-1} \bar{B} & \widetilde{A}^{N-2} \bar{B} & \bar{B}
\end{array}\right], \quad(8 \mathrm{a}) \\
\widetilde{\mathbf{Q}}:=\operatorname{diag}\left(\widetilde{Q}, \ldots, \widetilde{Q}, \widetilde{Q}_{\mathrm{r}}, Q_{f}\right), \mathbf{R}:=\operatorname{diag}\left(\bar{R}, \ldots, \bar{R}, \bar{R}_{\mathrm{r}}\right) .
\end{gathered}
$$

Here, the shifted matrices $\widetilde{A}:=\bar{A}-\bar{B} \bar{R}^{-1} \bar{S}^{\top}, \widetilde{A}_{\mathrm{r}}:=\bar{A}_{\mathrm{r}}-$ $\bar{B}_{\mathrm{r}} \bar{R}_{\mathrm{r}}^{-1} \bar{S}_{\mathrm{r}}^{\top}, \widetilde{Q}:=\bar{Q}-\bar{S} \bar{R}^{-1} \bar{S}^{\top}$ and $\widetilde{Q}_{\mathrm{r}}:=\bar{Q}_{\mathrm{r}}-\bar{S}_{\mathrm{r}} \bar{R}_{\mathrm{r}}^{-1} \bar{S}_{\mathrm{r}}^{\top}$ account for the cross terms in the cost function $(2 \mathrm{a})$. Finally, let the parameters for the constraints be

$$
\begin{aligned}
& Y:=E+G H^{-1} F^{\top}, \\
& G:=\left[\begin{array}{r}
I-\mathcal{R} \widetilde{\widetilde{B}} \\
-(I-\mathcal{R} \underline{\underline{B}}) \\
\underset{\widetilde{\mathbf{B}}}{\widetilde{\mathbf{B}}}
\end{array}\right], W:=\left[\begin{array}{r}
\hat{\mathbf{u}} \\
-\hat{\mathbf{u}} \\
-\hat{\mathbf{x}} \\
-\tilde{\mathbf{x}}
\end{array}\right], E:=\left[\begin{array}{r}
\mathcal{R} \widetilde{A} \\
-\mathcal{R} \\
-\widetilde{\vec{A}} \\
-\widetilde{\mathbf{A}} \\
\widetilde{\mathbf{A}}
\end{array}\right] .
\end{aligned}
$$

Consequently, the OCP can be written as

$$
\begin{aligned}
& \min _{\mathbf{z}} \ell_{z}\left(h, T, \mathrm{x}_{i}, \mathbf{z}\right):=\frac{1}{2}\|\mathbf{z}\|_{H(h, T)}^{2}+\frac{1}{2}\left\|\mathrm{x}_{i}\right\|_{L(h, T)}^{2} \\
& \text { s.t. } G \mathbf{z} \leq W+Y \mathrm{x}_{i} .
\end{aligned}
$$

This formulation is commonly used, e.g. in [4], except that $\mathbf{z}$ in (7) is modified slightly to accommodate for the cross terms associated with $\bar{S}$ and $\bar{S}_{\text {r }}$ in (2a). Further, the residual prediction step changes the constituents of the block matrices.

The value function and associated solution are

$$
\begin{aligned}
J_{z}\left(h, T, \mathbf{x}_{i}\right) & :=\min _{\mathbf{z}} \ell_{z}\left(h, T, \mathbf{x}_{i}, \mathbf{z}\right) \text { s.t. }(10 \mathrm{~b}) \\
& =J\left(h, T, \mathbf{x}_{i}\right) \text { given in }(3) \\
\mathbf{z}^{*}\left(h, T, \mathbf{x}_{i}\right) & :=\arg \min _{\mathbf{z}} \ell_{z}\left(h, T, \mathbf{x}_{i}, \mathbf{z}\right) \text { s.t. (10b). }
\end{aligned}
$$

$\mathbf{z}^{*}$ is the solution that minimises the value function. In the following, key properties of the OCP are highlighted to gain insight on how the optimality of a point changes as $h$ and $T$ are perturbed.

\section{Key properties of the system}

\subsection{Differentiability of transition and cost matrices}

The matrices $\bar{A}, \bar{B}, \bar{Q}, \bar{R}$ and $\bar{S}$ are differentiable w.r.t. $h$ and independent of $T$. The Taylor series expansion for the perturbation $h^{+}:=h+\delta_{h}$ as $\delta_{h} \rightarrow 0$ is

$$
\bar{A}^{+}:=\bar{A}\left(h^{+}\right)=\bar{A}(h)+\partial_{h} \bar{A}(h) \delta_{h}+\mathcal{O}\left(\delta_{h}^{2}\right)
$$

and similarly for $\bar{B}, \bar{Q}, \bar{R}$ and $\bar{S}$. The matrices $\bar{A}_{\mathrm{r}}$, $\bar{B}_{\mathrm{r}}, \bar{Q}_{\mathrm{r}}, \bar{R}_{\mathrm{r}}$ and $\bar{S}_{\mathrm{r}}$ are differentiable w.r.t. $h_{\mathrm{r}}$, thus differentiable w.r.t. $(h, T)$, for all $h_{\mathrm{r}} \in(0, h)$ since $h_{\mathrm{r}}:=T-N h$. 
Optimality is identified by the Karush-Kuhn-Tucker (KKT) conditions [12]. $\mathbf{z}^{*}$ is a solution to (10) iff

$$
\exists \boldsymbol{\mu}:\left\{\begin{array}{l}
-H \mathbf{z}^{*}=G^{\top} \boldsymbol{\mu} \\
\boldsymbol{\mu} \geq 0 \\
\mu_{j} c_{j}\left(\mathbf{z}^{*}\right)=0 \quad \forall j \in\left\{1, \ldots, n_{c}\right\} .
\end{array}\right.
$$

The solution is $\mathbf{z}^{*}=-H^{-1} G^{\top} \boldsymbol{\mu} . H$ is invertible as defined in $\S 2.2 . \boldsymbol{\mu}=:\left[\mu_{1} \cdots \mu_{n_{c}}\right]^{\top}$ contains the Lagrange multipliers associated with the constraints denoted by $\mathbf{c}(\mathbf{z}) \leq 0$ where $\mathbf{c}(\mathbf{z}):=G \mathbf{z}-W-Y \mathrm{x}_{i} \cdot c_{j}$ denotes the $j^{\text {th }}$ constraint i.e. $j^{\text {th }}$ row of $c(\mathbf{z})$.

The active set $\mathcal{A}\left(\mathbf{z}^{*}\right):=\left\{j \mid c_{j}\left(\mathbf{z}^{*}\right)=0\right\}$ contains the indices of the active constraints at the solution $\mathbf{z}^{*}$. Let the accent ' denotes correspondence to active constraints, e.g. $\boldsymbol{\mu}:=\left\{\mu_{j} \mid j \in \mathcal{A}\left(\mathbf{z}^{*}\right)\right\}, \dot{\boldsymbol{\mu}} \in \mathbb{R}^{n_{a}}$ and $n_{a}:=|\mathcal{A}|$ is the number of active constraints. From the complementarity condition (12c), elements of $\boldsymbol{\mu}$ for inactive constraints must be 0 , and those for the active constraints are

$$
\begin{aligned}
0=\dot{\mathbf{c}} & =\dot{G} \mathbf{z}^{*}-W^{\prime}-\dot{Y}^{\prime} \mathrm{x}_{i} \\
& =-\dot{G} H^{-1} G^{\top} \dot{\boldsymbol{\mu}}-W^{\prime}-\dot{Y} \mathrm{x}_{i} \\
\text { therefore, } \quad \dot{\boldsymbol{\mu}} & =-\left(\dot{G} H^{-1} \dot{G}^{\top}\right)^{-1}\left(\dot{W}+\dot{Y} \mathrm{x}_{i}\right) \\
\boldsymbol{\mu} & =\mathcal{I}_{\mathcal{A}\left(\mathbf{z}^{*}\right)} \dot{\mu} .
\end{aligned}
$$

The inverse in (13a) exists iff $\dot{G}$ is full rank. $\mathcal{I}_{\mathcal{A}} \in$ $\{0,1\}^{n_{c} \times n_{a}}$ is used to organise the Lagrange multipliers of the active constraint $\boldsymbol{\mu}$ into respective rows of $\boldsymbol{\mu}$.

\subsection{Feasibility}

Existence of the solution depends on the feasibility of the OCP, which in the context of designing $h$ and $T$ follows:

Definition 3 (Feasible design set $\mathcal{H}_{F}^{\mathrm{OCP}}\left(\mathrm{x}_{i}\right)$ of (2)) $\mathcal{H}_{F}^{\mathrm{OCP}}\left(\mathrm{x}_{i}\right)$ contains all $(h, T)$ for which the $O C P(2)$ is feasible given a current state $\mathrm{x}_{i}$.

In closed-loop, this notion is extended to recursive feasibility. This requires control invariance, i.e. for an initial state $x_{0}$ the MPC law (4) is feasible and keeps the system feasible at all subsequent sampling steps. This can be guaranteed with appropriately chosen $h$ and $T$ [9].

Definition 4 (Feasible design set $\mathcal{H}_{F}^{\mathrm{MPC}}\left(\mathrm{x}_{0}\right)$ of $(6)$ ) $\mathcal{H}_{F}^{\mathrm{MPC}}\left(\mathrm{x}_{0}\right)$ contains all $(h, T)$ such that the closed-loop system (6) is recursively feasible given an initial state $\mathrm{x}_{0}$.

\subsection{Uniqueness and non-degeneracy}

A sufficient condition for uniqueness is satisfaction of the linear independence constraint qualification (LICQ)
[12], achieved if the active constraint gradients are linearly independent, i.e. $G$ has full row rank so that the solution (13a) to the KKT conditions (12) is unique.

Definition 5 (Non-degenerate OCP) An OCP with solution $\mathbf{z}^{*}$ and active set $\mathcal{A}\left(\mathbf{z}^{*}\right)$ whose active constraint gradients $G$ satisfies LICQ is defined as non-degenerate.

Non-degeneracy can, for example, be guaranteed by removing state constraints.

Lemma 6 For a solution $\mathbf{z}^{*}$ of the OCP (10), if $\check{x}=$ $-\{1\}^{n_{x}} \infty, \hat{x}=\{1\}^{n_{x}} \infty$ and $\check{u}<\hat{u}$, then LICQ is satisfied.

PROOF. With no state constraints, $G$ constitutes only the upper two row blocks in $(9 \mathrm{~b})$. Linear dependence comes from row pairs $r$ and $r+(N+1) n_{u}$ for $r \in$ $\left\{1, \ldots, N n_{u}\right\}$, each with rows that are negative multiples of each other. These correspond to the upper and lowerbound for an input element that cannot both be active simultaneously. Therefore the active constraint gradients in the rows of $G$ must be linearly independent.

\subsection{Continuity of the OCP solution}

The residual sampling interval $h_{\mathrm{r}}$ ensures that the OCP solution remains continuous across changing $N$.

Lemma 7 (Continuity of $\mathbf{z}^{*}$ at $T / h \in \mathbb{N}_{>0}$ ) Consider an OCP (2) that is non-degenerate per Definition 5, given an $\mathrm{x}_{i} \in \mathbb{R}^{n_{x}}$ such that $(h, T) \in \mathcal{H}_{F}^{\mathrm{OCP}}\left(\mathrm{x}_{i}\right) \neq \emptyset$. The unique optimal solution $(h, T) \mapsto \mathbf{z}^{*}\left(h, T, \mathrm{x}_{i}\right)$ of the OCP is a continuous function of $(h, T)$ at $T / h \in \mathbb{N}_{>0}$ i.e. at boundaries where $N$ changes.

PROOF. An OCP (2) with $N$ prediction steps and $h_{\mathrm{r}}=0$ has the same solution $\mathbf{z}^{*}$ as that with $N-1$ steps and $h_{\mathrm{r}}=h$, relying on the uniqueness of $\mathbf{z}^{*}(\S 3.4)$. $\mathbf{z}^{*}$ has effectively the same dimension $N$ for both cases. Hence, $\mathbf{z}^{*}$ must be continuous at the $N$ to $(N-1)$ boundary.

\subsection{Stability of the closed-loop system}

The closed-loop value function (6a) is an infinite series. For it to be finite-valued, it has to be convergent, which can be guaranteed by stability of the closed-loop system.

Definition 8 (Stable design set $\mathcal{H}_{S}^{\mathrm{MPC}}\left(\mathrm{x}_{0}\right)$ of $(6)$ ) $\mathcal{H}_{S}^{\mathrm{MPC}}\left(\mathrm{x}_{0}\right) \subseteq \mathcal{H}_{F}^{\mathrm{MPC}}\left(\mathrm{x}_{0}\right)$ contains all $(h, T)$ such that the closed-loop system (6) has a region of attraction containing the initial state $\mathrm{x}_{0}$ as well as an origin that is reachable and exponentially stable.

Lemma 9 (Exponential stability of $(2 \mathbf{a}-\mathbf{c}))$ If $(\bar{A}, \bar{B})$ is reachable, and $\left[\begin{array}{cc}\bar{Q} & \bar{S} \\ \bar{S}^{\top} & \bar{R}\end{array}\right] \geq 0$ and $\left[\begin{array}{ll}\bar{Q}_{r} & \bar{S}_{r} \\ \bar{S}_{r}^{\top} & \bar{R}_{r}\end{array}\right] \geq 0$, then the origin of the unconstrained system $(2 \mathrm{a}-\mathrm{c})$ is exponentially stable.

PROOF. The proof follows Theorem 4.1 in [6]. 
Remark 10 In Lemma 9, the first condition requires that $(A, B)$ is reachable, and $h \neq 2 \pi n / \Im\left(\lambda_{i}(A)-\lambda_{j}(A)\right)$, $\forall i, j \in\left\{1, \ldots, n_{x}\right\}, i \neq j, n \in \mathbb{N}_{>0}$ [8] when $A$ has complex eigenvalues. Let $\mathcal{H}_{\mathrm{C}}$ contain all of these 'critical' sampling periods. The second condition is equivalent to a positive semidefinite stage cost, which has been guaranteed by $Q \geq 0, R>0$. It follows that $\mathcal{H}_{S}^{\mathrm{MPC}}\left(\mathrm{x}_{0}\right) \subseteq \mathcal{H}_{F}^{\mathrm{MPC}}\left(\mathrm{x}_{0}\right) \backslash\left\{(h, T) \mid h \in \mathcal{H}_{\mathrm{C}}\right\}$. Stability of the full system (2) is discussed in e.g. [10].

Lemma 11 For $(h, T) \in \mathcal{H}_{S}^{\mathrm{MPC}}\left(\mathrm{x}_{0}\right)$, the closed-loop value function $V\left(\cdot, \cdot, \mathrm{x}_{0}\right)$ in (6) is a convergent series.

PROOF. $(h, T) \in \mathcal{H}_{S}^{\mathrm{MPC}}\left(\mathrm{x}_{0}\right) \Rightarrow$ exponential stability of (6). Thus, the ratio test [11] on (5) yields $\lim _{i \rightarrow \infty} \frac{\ell_{0}\left(h, T, \mathrm{x}_{i+1}\right)}{\ell_{0}\left(h, T, \mathrm{x}_{i}\right)}=\rho<1$. Therefore, $V$ is a convergent series and finite-valued in the limit $i \rightarrow \infty$.

\section{Smoothness properties of the value function}

\subsection{Open-loop}

The following analysis considers the optimal control at a given sampling instant w.r.t. sampling and prediction horizon times. This extends investigations such as [5] that solely analyse sampling time.

In subsequent analyses, non-degeneracy of the KKT conditions is assumed.

Assumption 12 (Non-degeneracy of the OCP (2)) If the equivalent $O C P(10)$ is non-degenerate per Definition 5, then the OCP (2) is non-degenerate.

To show smoothness properties, a perturbation $h^{+}:=$ $h+\delta_{h}$ and/or $T^{+}:=T+\delta_{T}$ as $\delta_{h} \rightarrow 0$ and/or $\delta_{T} \rightarrow 0$ is performed. Consider when the perturbation does not change $N=\lfloor T / h\rfloor$. Let the perturbed optimal solution be $\mathbf{z}^{+}$, with perturbed Lagrange multipliers $\boldsymbol{\mu}^{+}$and perturbed value function $J_{z}^{+}$. To prove that $J_{z}$ is differentiable, the differentiability of the Lagrange multipliers and OCP solution are first considered.

Lemma 13 (Differentiability of $\boldsymbol{\mu}$ ) The Lagrange multipliers $\boldsymbol{\mu}$ are differentiable w.r.t. $(h, T)$ for a nondegenerate OCP (10) per Definition 5 and a given $N$.

PROOF. The proof is given in Appendix A.

Lemma 14 (Differentiability of $\mathbf{z}^{*}$ ) For a nondegenerate OCP (10) per Definition 5, the solution $\mathbf{z}^{*}$ is differentiable w.r.t. $(h, T)$ for a given $N$. Guarantees of differentiability are lost across changes in $N$.

PROOF. The proof is given in Appendix B.

Knowing the differentiability of the Lagrange multipliers and solution to the OCP, the main result of the differentiability of the open-loop value function can be stated.
Theorem 15 (Continuity of $J$ ) Consider the $O C P(2)$, given an $\mathrm{x}_{i} \in \mathbb{R}^{n_{x}}$ such that $(h, T) \in \mathcal{H}_{F}^{\mathrm{OCP}}\left(\mathrm{x}_{i}\right) \neq \emptyset$ and Assumption 12 is satisfied. The open-loop value function $(h, T) \mapsto J\left(h, T, \mathrm{x}_{i}\right)$ is a

- continuous function of $(h, T)$,

- differentiable function of $(h, T)$ for a fixed $N:=\lfloor h / T\rfloor$.

PROOF. Consider the value function $J_{z}\left(h, T, \mathrm{x}_{i}\right)(11 \mathrm{a})$ of problem (10) at a solution $\mathbf{z}^{*}$, with $\boldsymbol{\mu}$ satisfying the KKT conditions (12). Consider a perturbation $h^{+}:=h+$ $\delta_{h}$ as $\delta_{h} \rightarrow 0$ and $T^{+}:=T+\delta_{T}$ as $\delta_{T} \rightarrow 0$ that does not change $N=\mid T / h\rfloor$. From Lemma 14, and satisfaction of Assumption 12, the value function can be expressed as

$$
\begin{aligned}
& J_{z}\left(h^{+}, T^{+}, \mathbf{x}_{i}\right) \\
& =\ell_{z}^{+}\left(\mathbf{z}^{+}\right)+\partial_{h} \ell_{z}\left(\mathbf{z}^{+}\right) \delta_{h}+\partial_{T} \ell_{z}\left(\mathbf{z}^{+}\right) \delta_{T}+\mathcal{O}\left(\delta_{h}^{2}, \delta_{T}^{2}\right) \\
& =\left\|\mathbf{z}^{*}+\partial_{h} z \delta_{h}+\partial_{T} z \delta_{T}\right\|_{H+\partial_{h} H \delta_{h}+\partial_{T} H \delta_{T}}^{2}+ \\
& \quad\left\|\mathbf{x}_{i}\right\|_{L+\partial_{h} L \delta_{h}+\partial_{T} L \delta_{T}}^{2}+\mathcal{O}\left(\delta_{h}^{2}, \delta_{T}^{2}\right) \\
& =J_{z}\left(h, T, \mathbf{x}_{i}\right)+\partial_{h} J_{z} \delta_{h}+\partial_{T} J_{z} \delta_{T}+\mathcal{O}\left(\delta_{h}^{2}, \delta_{T}^{2}\right)
\end{aligned}
$$

for some finite $\partial_{h} J_{z}(h, T)$ and $\partial_{T} J_{z}(h, T)$. From (11b), $J_{z}\left(h, T, x_{i}\right)=J\left(h, T, x_{i}\right)$. Hence, the value function $J$ is differentiable, thus also continuous w.r.t. $(h, T)$ for a fixed $N$. At boundaries where $N$ changes, continuity of the value function is implied by Lemma 7 .

To illustrate the results, consider a harmonic oscillator

$$
\dot{x}=\left[\begin{array}{cc}
0 & \omega \\
-\omega & 0
\end{array}\right] x+\left[\begin{array}{l}
0 \\
1
\end{array}\right] u
$$

with $\omega=1$. Following a number of trials on different plant models, the harmonic oscillator is chosen as it helps to best demonstrate the theoretical results numerically. The cost weights are chosen as

$$
Q=\left[\begin{array}{ll}
3 & 0 \\
0 & 2
\end{array}\right], \quad R=1, \quad Q_{f}=\begin{aligned}
& \text { as specified in } \\
& \text { figure captions. }
\end{aligned}
$$

If no state constraint is imposed, non-degeneracy is guaranteed (Lemma 6). An illustrative case is given in Fig. 1. The open-loop value-function remains continuous and appears differentiable with changing active set and nondifferentiable across changes in $N$, as per Theorem 15 . Non-monotonicity w.r.t. $(h, T)$ is observed, particularly more often at small sampling times.

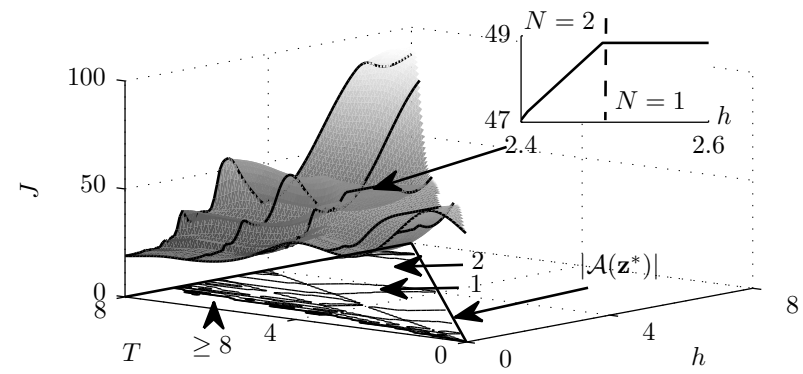

Fig. 1. Open-loop value function $J$ for the harmonic oscillator (14) with the $\operatorname{OCP}(2) .|\mathrm{u}| \leq \frac{1}{4}, \mathrm{x}_{i}=\left[\begin{array}{ll}1 & 1\end{array}\right]^{\top}, Q_{f}=10 Q$. The contour of $\left|\mathcal{A}\left(\mathbf{z}^{*}\right)\right|$ is plotted on the $z$-plane. 


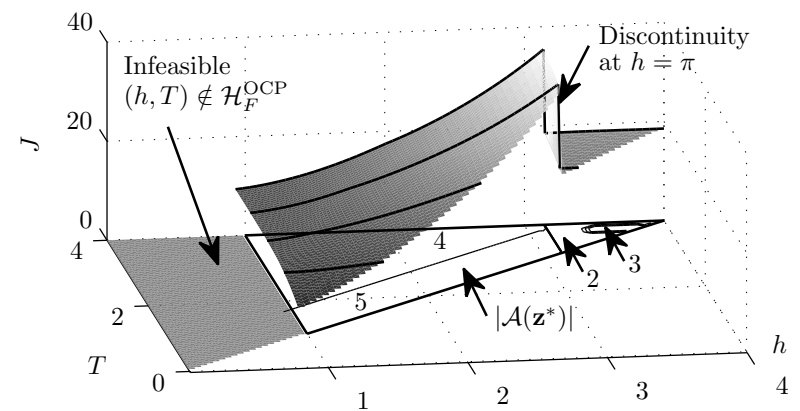

Fig. 2. Open-loop value function $J$ for the harmonic oscillator (14) with the $\operatorname{OCP}(2) .|\mathrm{x}| \leq 1,|\mathrm{u}| \leq 1, \mathrm{x}_{i}=\left[\begin{array}{ll}1 & 1\end{array}\right]^{\top}$, $Q_{f}=0$. The contour of $\left|\mathcal{A}\left(\mathbf{z}^{*}\right)\right|$ is plotted on the $z$-plane.

The problem is degenerate if state constraints are imposed (§3.4). Solution to the KKT conditions is nonunique, inconsistent with (A.1). Consequently, continuity is not guaranteed anymore across active set changes, as seen in Fig. 2. Discontinuity happens at $h=\pi$, where the size of the active set changes to 2 from either 5 or 4 . The loss of guarantee is consistent with the results in [3]. The figure also demonstrates the region associated with the infeasible design set $(h, T) \notin \mathcal{H}_{F}^{\mathrm{OCP}}\left(\mathrm{x}_{i}\right)$.

\subsection{Closed-loop}

In control applications, the closed-loop value function is a true measure of performance. Smoothness properties of the closed-loop value function can be established following the results for the open-loop case.

\section{Lemma 16 (Differentiability of state transition)}

The transition of the state (6b) of the closed-loop system (6) is differentiable w.r.t. $(h, T)$ for a given $N$.

PROOF. Consider the state transition at time $i=0$; $\mathrm{x}_{1}=\bar{A} \mathrm{x}_{0}+\bar{B} u_{0}^{*}\left(h, T, \mathrm{x}_{0}\right)$. The OCP solution is differentiable w.r.t. $(h, T)$ for a given $N$ as per Lemma 14 , hence also the optimal input. That is, the perturbation $h^{+}:=h+\delta_{h}$ as $\delta_{h} \rightarrow 0$ yields $u_{0}^{*+}\left(\mathrm{x}_{i}\right)=u_{0}^{*}\left(\mathrm{x}_{i}\right)+$ $\partial_{h} u_{0}^{*}\left(\mathrm{x}_{i}\right) \delta_{h}+\mathcal{O}\left(\delta_{h}^{2}\right)$ and

$$
\begin{aligned}
\mathrm{x}_{1}^{+}= & \left(\bar{A}+\partial_{h} \bar{A} \delta_{h}\right) \mathrm{x}_{0}+ \\
& \left(\bar{B}+\partial_{h} \bar{B} \delta_{h}\right)\left(u_{0}^{*}\left(\mathrm{x}_{0}\right)+\partial_{h} u_{0}^{*}\left(\mathrm{x}_{0}\right) \delta_{h}\right)+\mathcal{O}\left(\delta_{h}^{2}\right) \\
= & \mathrm{x}_{1}+\partial_{h} \mathrm{x}_{1} \delta_{h}+\mathcal{O}\left(\delta_{h}^{2}\right) .
\end{aligned}
$$

So, at each sampling instant after the initial, $\mathrm{x}_{i}^{+}=\mathrm{x}_{i}+$ $\partial_{h} \mathrm{x}_{i} \delta_{h}+\mathcal{O}\left(\delta_{h}^{2}\right), \forall i \in \mathbb{N}_{>0}$. A perturbation on $T$ affects the system similarly.

Consider now the properties of the term $\ell_{0}$ for the closedloop value function $V$ (6a), following Theorem 15 .

Lemma 17 (Differentiability of $\ell_{0}$ ) Consider the $O C P(2)$, given an $\mathrm{x}_{i} \in \mathbb{R}^{n_{x}}$ such that $(h, T) \in$ $\mathcal{H}_{F}^{\mathrm{OCP}}\left(\mathrm{x}_{i}\right) \neq \emptyset$ and Assumption 12 is satisfied. The first element of the cost function $(h, T) \mapsto \ell_{0}\left(h, T, \mathrm{x}_{i}\right)$ is differentiable w.r.t. $(h, T)$ for a fixed $N$.
PROOF. For a perturbation $h^{+}:=h+\delta_{h}$ as $\delta_{h} \rightarrow 0$ which does not change $N, \ell_{0}(5)$ is expressed as $\ell_{0}^{+}:=$ $\ell_{0}\left(h^{+}\right)=\left\|\left[\begin{array}{c}x_{i}^{+} \\ u_{0}^{*+}\left(x_{i}\right)\end{array}\right]\right\|^{2}\left[\begin{array}{cc}\bar{Q}^{+} & \bar{S}^{+} \\ \bar{S}^{+\top} & \bar{R}^{+}\end{array}\right]$for some perturbed cost weights $\bar{Q}^{+}, \bar{R}^{+}$and $\bar{S}^{+}$, current state $\mathrm{x}_{i}^{+}$, and optimal input $u_{0}^{*+}\left(\mathrm{x}_{i}\right)$. The cost weights are differentiable; $\bar{Q}^{+}=$ $\bar{Q}+\partial_{h} \bar{Q} \delta_{h}+\mathcal{O}\left(\delta_{h}^{2}\right)$ etc. Lemma 16 states that the current state and optimal input are differentiable. Hence, the perturbed value of $\ell_{0}$ can be expressed as

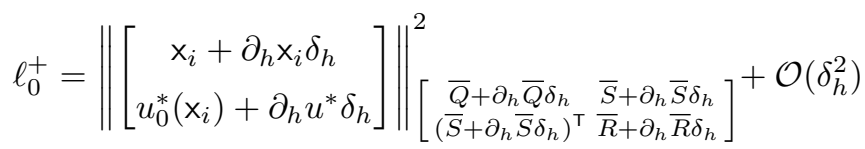

$$
\begin{aligned}
& =\ell_{0}\left(h, x_{i}\right)+\partial_{h} \ell_{0} \delta_{h}+\mathcal{O}\left(\delta_{h}^{2}\right) \text {. }
\end{aligned}
$$

A perturbation in $T$ affects the system similarly.

Theorem 18 (Continuity of $V$ ) Consider the $M P C$ system (6), given an $\mathrm{x}_{0} \in \mathbb{R}^{n_{x}}$ such that $(h, T) \in$ $\mathcal{H}_{S}^{\mathrm{MPC}}\left(\mathrm{x}_{0}\right) \neq \emptyset$, controlled by (4) from an $O C P(2)$ satisfying Assumption 12. The closed-loop value function $(h, T) \mapsto V\left(h, T, \mathrm{x}_{0}\right)$ is a

- continuous function of $(h, T)$,

- differentiable function of $(h, T)$ for a fixed $N:=\lfloor h / T\rfloor$.

PROOF. The closed-loop value function (6a) is a sum of the terms $\ell_{0}$ so that $V^{+}:=V\left(h^{+}, T^{+}, \mathrm{x}_{0}\right)=$ $\ell_{0}\left(h^{+}, T^{+}, \mathrm{x}_{0}\right)+\sum_{i=1}^{\infty} \ell_{0}\left(h^{+}, T^{+}, \mathrm{x}_{i}^{+}\right)$. For $(h, T) \in$ $\mathcal{H}_{S}^{\mathrm{MPC}}\left(\mathrm{x}_{0}\right)$, the summation series would be convergent (finite-valued) by Lemma 11. From Lemmas 16 and 17, $V^{+}$can be expanded to yield

$$
V^{+}=V\left(h, T, \mathrm{x}_{0}\right)+\partial_{h} V \delta_{h}+\partial_{T} V \delta_{T}+\mathcal{O}\left(\delta_{h}^{2}, \delta_{T}^{2}\right) .
$$

for some finite $\partial_{h} V(h, T)$ and $\partial_{T} V(h, T)$. Therefore, the value function $V$ is differentiable w.r.t. $(h, T)$ for a fixed $N$. At boundaries where $N$ changes, continuity of the value function is implied by Lemma 7 .

Remark 19 A linear combination of the value function $V\left(h, T, \mathrm{x}_{0}\right)$ (or $\left.J\left(h, T, \mathrm{x}_{i}\right)\right)$ for different initial states $\mathrm{x}_{0}$ (or $\mathrm{x}_{i}$ ) preserves the smoothness properties presented.

So far, control performance is measured by the value function for a given initial condition. A more comprehensive measure that considers multiple initial conditions can be easily obtained (Remark 19).

Fig. 3 plots the closed-loop value function for the harmonic oscillator $(14,15)$ and $Q_{f}=P_{\text {DARE }}$ is the solution of the discrete algebraic Riccati equation, chosen to emulate an infinite horizon cost. The function is continuous as per Theorem 18. Non-differentiability at chang$\operatorname{ing} N$ is observed. The value function becomes very large around $h=\pi$, as $\pi \in \mathcal{H}_{\mathrm{C}}$ is a critical sampling period for the system (14) [8]. The discrete plant $(\bar{A}, \bar{B})$ loses full controllability and is unstable, as $\{(h, T) \mid h=\pi\} \notin$ $\mathcal{H}_{S}^{\mathrm{MPC}}\left(\mathrm{x}_{0}\right)$ (Remark 10). At these points the value function $V_{\mathrm{u}}$ becomes unbounded and continuity is lost. 


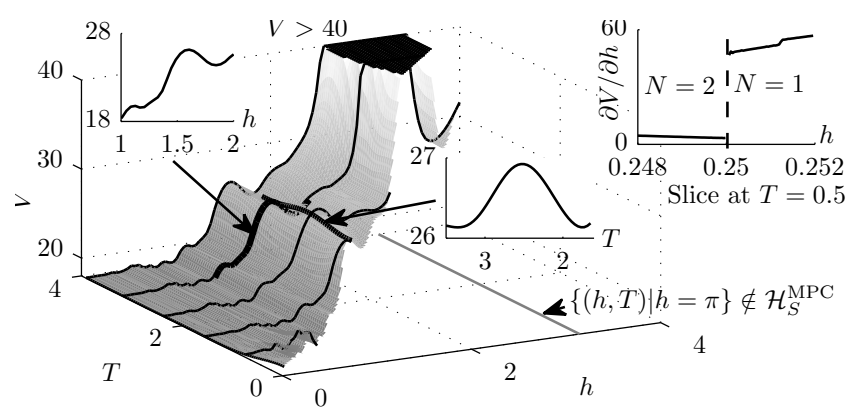

Fig. 3. Closed-loop value function $V$ for the harmonic oscillator (14) with the OCP (2). $|\mathbf{u}| \leq \frac{1}{4}, \mathrm{x}_{0}=\left[\begin{array}{ll}1 & 1\end{array}\right]^{\top}, Q_{f}=P_{\mathrm{DARE}}$, $N_{\text {sim }}=250 . z$-axis is truncated for clarity.

One might expect that longer prediction horizons and/or shorter sampling intervals are always desirable; a longer prediction horizon retrieves more future information while a shorter sampling interval gives better information resolution and more responsiveness, since control actions can be applied at a higher rate. However, Fig. 3 demonstrates that these expectations are not necessarily upheld. It shows that there are multiple local minima on the surface w.r.t. both sampling interval and prediction horizon, particularly at smaller sampling times. The non-monotonicity is made clear by the subplots showing slices for a given $h$ or $T$. Such a property is also observed in the open-loop case w.r.t. $h$ (Fig. 1$)$.

\section{Conclusions and future work}

We have investigated the smoothness properties of the MPC value function in open- and closed-loop as performance measures of constrained linear systems. The main results presented are: (1) The open- and closedloop value functions are continuous w.r.t. sampling and horizon times, as well as differentiable for a given number of prediction steps, if the OCP is non-degenerate. If the OCP is degenerate, the guarantee of continuity is lost, as demonstrated numerically. (2) The open- and closedloop value functions are non-monotonic w.r.t. sampling period and prediction horizon, as shown numerically.

The control architecture considered in this paper is an MPC scheme with linear constraints and prediction model. For future work, typical extensions could include a nonlinear terminal constraint or nonlinear prediction model for the purpose of guaranteeing stability and better control performance [2]. With these extensions, the problem might involve more complex KKT conditions.

The body of work presented in the paper is useful in the context of developing systematic methods for the selection of optimal sampling and prediction times for MPC. Such approaches, for example, would seek an optimal trade-off between closed-loop performance and computational effort, as well as be able to handle non-differentiability and multiple optima, i.e. nonmonotonicity on the optimisation surface.

\section{References}

[1] V. Bachtiar, E. C. Kerrigan, W. Moase, and C. Manzie. Smoothness properties of the MPC value function in open and closed-loop with respect to sampling time and prediction horizon. In IEEE Asian Contr Conf P, 2015.

[2] V. Bachtiar, T. Mühlpfordt, W. Moase, T. Faulwasser, R. Findeisen, and C. Manzie. Nonlinear model predictive missile control with a stabilising terminal constraint. In IFAC World Congress P, 2014.

[3] M. Baotic, F. Borrelli, A. Bemporad, and M. Morari. Efficient on-line computation of constrained optimal control. SIAM J Contr and Optim, 47(5):2470-2489, 2008.

[4] A. Bemporad, M. Morari, V. Dua, and E. N. Pistikopoulos. The explicit linear quadratic regulator for constrained systems. Automatica, 38(1):3-20, 2002.

[5] E. Bini and G. M. Buttazzo. The optimal sampling pattern for linear control systems. IEEE Trans Automatic Control, 59(1):78-90, 2014.

[6] S. W. Chan, G. C. Goodwin, and K. S. Sin. Convergence properties of the Riccati difference equation in optimal filtering of nonstabilizable systems. IEEE Trans Automatic Control, 29(2):110-118, 1984.

[7] F. Di Palma and L. Magni. On optimality of nonlinear model predictive control. Systems \& Control Ltr, 56(1):58-61, 2007.

[8] A. H. Levis, R. A. Schlueter, and M. Athans. On the behaviour of optimal linear sampled-data regulators. Int $J$ of Control, 13(2):343-361, 1971.

[9] D. Q. Mayne. Model predictive control: Recent developments and future promise. Automatica, 50(12):2967-2986, 2014.

[10] J. B. Rawlings and K. R. Muske. The stability of constrained receding horizon control. IEEE Trans Automatic Control, 38(10):1512-1516, 1993.

[11] J. Stewart. Calculus. Cengage Learning, 7 edition, 2011.

[12] S. Wright and J. Nocedal. Numerical optimization. Springer, NY, 1999.

\section{A Proof of Lemma 13}

This proof will show that the Lagrange multipliers can be expressed in the form

$$
\boldsymbol{\mu}^{+}=\boldsymbol{\mu}^{*}+\partial_{h} \mu \delta_{h}+\partial_{T} \mu \delta_{T}+\mathcal{O}\left(\delta_{h}^{2}, \delta_{T}^{2}\right)
$$

for some finite $\partial_{h} \mu$ and $\partial_{T} \mu$, showing the differentiability of $\boldsymbol{\mu}$. Consider a perturbation $h^{+}=h+\delta_{h}$ as $\delta_{h} \rightarrow 0$.

Case $1\left(\mathcal{A}\left(\mathbf{z}^{*}\right)=\mathcal{A}\left(\mathbf{z}^{+}\right)\right)$If the active set stays the same, the perturbed KKT conditions have a solution of $\dot{\boldsymbol{\mu}}^{+}=$ $-\left(\left(\dot{G}+\partial_{h} \dot{G} \delta_{h}\right)\left(H+\partial_{h} H \delta_{h}\right)^{-1}\left(\dot{G}+\partial_{h} \dot{G} \delta_{h}\right)^{\top}\right)^{-1}(\dot{W}+$ $\left.\left(\dot{Y}+\partial_{h} \dot{Y} \delta_{h}\right) x_{i}\right)=\dot{\mu}^{*}+\partial_{h} \dot{\mu} \delta_{h}+\mathcal{O}\left(\delta_{h}^{2}\right)$ for some finite $\partial_{h} \mu$, instead of (13a). This is organised as follows:

$$
\begin{aligned}
& \boldsymbol{\mu}^{+}=\mathcal{I}_{\mathcal{A}\left(\mathbf{z}^{+}\right)} \dot{\boldsymbol{\mu}}^{+}=\mathcal{I}_{\mathcal{A}\left(\mathbf{z}^{*}\right)}\left(\dot{\boldsymbol{\mu}}^{*}+\partial_{h} \dot{\mu} \delta_{h}+\mathcal{O}\left(\delta_{h}^{2}\right)\right) \\
& =\boldsymbol{\mu}^{*}+\partial_{h} \mu \delta_{h}+\mathcal{O}\left(\delta_{h}^{2}\right) \text {. }
\end{aligned}
$$

Case $2\left(\mathcal{A}\left(\mathbf{z}^{*}\right) \subset \mathcal{A}\left(\mathbf{z}^{+}\right)\right)$Consider a perturbation causing a constraint $e \notin \mathcal{A}\left(\mathbf{z}^{*}\right)$ to be active at some point 
along the perturbation, $\mathcal{A}\left(\mathbf{z}^{*}\right) \subset \mathcal{A}\left(\mathbf{z}^{+}\right)=\mathcal{A}\left(\mathbf{z}^{*}\right) \cup\{e\}$. The multipliers $\boldsymbol{\mu}^{+}$as constraint $e$ activates are

$$
\begin{aligned}
& \dot{\boldsymbol{\mu}}^{+}=\left[\begin{array}{c}
\hat{\mu}_{o}^{+} \\
\dot{\mu}_{e}^{+}
\end{array}\right]=\left(\left\|\left[\begin{array}{c}
G+\partial_{h} G \delta_{h} \\
\dot{G}_{e}^{+}
\end{array}\right]^{\top}\right\|_{\left(H+\partial_{h} H \delta_{h}\right)^{-1}}^{2}\right)^{-1} \\
& {\left[\begin{array}{c}
\hat{W}+\left(\hat{Y}+\partial_{h} \hat{Y} \delta_{h}\right) x_{i} \\
\hat{W}_{e}^{+}+\hat{Y}_{e}^{+} x_{i}
\end{array}\right]+\mathcal{O}\left(\delta_{h}^{2}\right)} \\
& =\left[\begin{array}{cc}
\dot{G} H^{-1} \dot{G}^{\mathrm{T}} & \dot{G}^{-1} \dot{G}_{e}^{+\mathrm{\top}} \\
\dot{G}_{e}^{+} H^{-1} \dot{G}^{\mathrm{T}} & \dot{G}_{e}^{+} H^{-1} \dot{G}_{e}^{+\top}
\end{array}\right]^{-1}\left[\begin{array}{c}
\hat{W}+\dot{Y} \mathrm{x}_{i} \\
\dot{W}_{e}^{+}+\dot{Y}_{e}^{+} \mathrm{x}_{i}
\end{array}\right] \\
& +\left[\begin{array}{l}
\partial_{h} \dot{\mu}_{o} \\
\partial_{h} \tilde{\mu}_{e}
\end{array}\right] \delta_{h}+\mathcal{O}\left(\delta_{h}^{2}\right)
\end{aligned}
$$

for some finite $\partial_{h} \dot{G}, \partial_{h} \dot{\mu}_{o}$ and $\partial_{h} \dot{\mu}_{e}$. The subscript $e$ denotes rows of $\dot{G}, \dot{W}$, etc. associated with the entering constraint, and $o$ for the original constraint set. Let $\left[\begin{array}{cc}\dot{G} H^{-1} \dot{G}^{\top} & \dot{G} H^{-1} \dot{G}_{e}^{+\top} \\ \dot{G}_{e}^{+} H^{-1} \dot{G}^{\top} & \dot{G}_{e}^{+} H^{-1} \dot{G}_{e}^{+\top}\end{array}\right]=\left[\begin{array}{cc}\mathcal{G}_{1} & \mathcal{G}_{2} \\ \mathcal{G}_{3} & \mathcal{G}_{4}\end{array}\right]=: \mathcal{G}$ and the Schur's complement $\mathcal{G}_{\mathrm{S}}:=\mathcal{G}_{4}-\mathcal{G}_{3} \mathcal{G}_{1}^{-1} \mathcal{G}_{2}$. The solution to (A.2) can be found first by block inversion of $\mathcal{G}$.

First, the lower block can be expressed as $\dot{\mu}_{e}^{+}=$ $-\mathcal{G}_{\mathrm{S}}^{-1}\left(\mathcal{G}_{3} \mathcal{G}_{1}^{-1}\left(\dot{W}+\dot{Y} \mathrm{x}_{i}\right)-\dot{W}_{e}^{+}-\dot{Y}_{e}^{+} \mathrm{x}_{i}\right)+\partial_{h} \dot{\mu}_{e} \delta_{h}+\mathcal{O}\left(\delta_{h}^{2}\right)$. After some algebra, expansion of the bracketed terms above would reveal that $\mathcal{G}_{3} \mathcal{G}_{1}^{-1}\left(\hat{W}+\dot{Y} \mathrm{x}_{i}\right)-\hat{W}_{e}^{+}-\hat{Y}_{e}^{+} \mathrm{x}_{i}=$ $0+\partial_{h} \mathcal{G}_{e} \delta_{h}+\mathcal{O}\left(\delta_{h}^{2}\right)$. This consistent with the fact that $\mu_{e}^{*}=0$ since the constraint $e$ is inactive in the unperturbed OCP i.e. $c_{e}\left(\mathbf{z}^{*}\right)<0$. Substitution yields the Lagrange multiplier for the newly active constraint $\hat{\mu}_{e}^{+}=\left(\partial_{h} \dot{\mu}_{e}+\partial_{h} \mathcal{G}_{e}\right) \delta_{h}+\mathcal{O}\left(\delta_{h}^{2}\right)$.

For the upper block, values are obtained by substituting (13a) and the bracket expansion above after the blockwise inversion, yielding $\mu_{o}^{+}=\dot{\boldsymbol{\mu}}^{*}+\left(\partial_{h} \dot{\mu}_{o}+\partial_{h} \mathcal{G}_{o}\right) \delta_{h}+$ $\mathcal{O}\left(\delta_{h}^{2}\right)$. Substituting these into (A.2) yields

$$
\begin{aligned}
\boldsymbol{\mu}^{+}=\mathcal{I}_{\mathcal{A}\left(\mathbf{z}^{+}\right)} \dot{\boldsymbol{\mu}}^{+}=\boldsymbol{\mu}^{*}+ & \left(\mathcal{I}_{\mathcal{A}\left(\mathbf{z}^{*}\right)}\left(\partial_{h} \mu_{o}+\partial_{h} \mathcal{G}_{o}\right)+\right. \\
& \left.\mathcal{I}_{\{e\}}\left(\partial_{h} \mu_{e}+\partial_{h} \mathcal{G}_{e}\right)\right) \delta_{h}+\mathcal{O}\left(\delta_{h}^{2}\right) .
\end{aligned}
$$

Case $3\left(\mathcal{A}\left(\mathbf{z}^{*}\right) \supset \mathcal{A}\left(\mathbf{z}^{+}\right)\right)$To keep the working tidy, suppose that the exiting constraint is located at the end of the set $\mathcal{A}\left(\mathbf{z}^{*}\right)$. The perturbed solution is now $\dot{\mu}^{+}=\mathcal{I}_{\mathcal{A}\left(\mathbf{z}^{*}\right)}\left(\left\|\left(\dot{G}+\partial_{h} G \delta_{h}\right)^{\top}\right\|_{\left(H+\partial_{h} H \delta_{h}\right)^{-1}}^{2}\right)^{-1}(\dot{W}+(\dot{Y}+$ $\left.\left.\partial_{h}^{Y} \delta_{h}\right) \mathrm{x}_{i}\right)-\mathcal{I}_{\{e\}}\left(\left\|\left(\dot{G}_{e}+\partial_{h} G_{e} \delta_{h}\right)^{\top}\right\|_{\left(H+\partial_{h} H \delta_{h}\right)^{-1}}^{2}\right)^{-1}\left(\dot{W}_{e}+\right.$ $\left.\left(\dot{Y}_{e}+\partial_{h} Y_{e} \delta_{h}\right) \mathrm{x}_{i}\right)$, where the subtractive term is to make the Lagrange multipliers associated with the exiting constraint zero. Further expansion yields

$$
\begin{aligned}
\dot{\boldsymbol{\mu}}^{+}= & \mathcal{I}_{\mathcal{A}\left(\mathbf{z}^{*}\right)}\left(\dot{G} H^{-1} \dot{G}^{\top}\right)^{-1}\left(\dot{W}+\dot{Y} \mathrm{x}_{i}\right)- \\
& \mathcal{I}_{\{e\}}\left(\dot{G}_{e} H^{-1} \dot{G}_{e}^{\top}\right)^{-1}\left(\dot{W}_{e}+\dot{Y}_{e} \mathrm{x}_{i}\right)+\partial_{h} \mu \delta_{h}+\mathcal{O}\left(\delta_{h}^{2}\right) \\
= & {\left[\begin{array}{ll}
\mathcal{I}_{\mathcal{A}\left(\mathbf{z}^{+}\right)} & 0
\end{array}\right] \dot{\boldsymbol{\mu}}^{*}+\partial_{h} \dot{\mu} \delta_{h}+\mathcal{O}\left(\delta_{h}^{2}\right) . }
\end{aligned}
$$

\section{B Proof of Lemma 14}

Case 1 (Constant $N$ ) Consider a perturbation $h^{+}:=$ $h+\delta_{h}$ as $\delta_{h} \rightarrow 0$ and that, over the perturbation, the number of prediction steps remains constant. Subject to this, $H\left(h^{+}\right)=H(h)+\partial_{h}^{H} \delta_{h}+\mathcal{O}\left(\delta_{h}^{2}\right)$ and similarly for $L, G, W$ and $Y$ as these matrices consist of addition, multiplication and/or inversion of the differentiable $\bar{A}$, $\bar{B}, \bar{Q}, \bar{R}, \bar{S}, \bar{Q}_{\mathrm{r}}, \bar{R}_{\mathrm{r}}$ and/or $\bar{S}_{\mathrm{r}}$. The perturbed OCP is

$$
\mathbf{z}^{*+}\left(\mathrm{x}_{i}\right)=\arg \min _{\mathbf{z}} \ell_{z}^{+}\left(\mathbf{z}, h^{+}\right)
$$

where $\ell_{z}^{+}\left(\mathbf{z}, h^{+}\right):=\|\mathbf{z}\|_{H+\partial_{h} H \delta_{h}}^{2}+\left\|\mathbf{x}_{i}\right\|_{L+\partial_{h} L \delta_{h}}^{2}+\mathcal{O}\left(\delta_{h}^{2}\right)=$ $\ell_{z}(\mathbf{z})+\partial_{h} \ell_{z}(\mathbf{z}) \delta_{h}+\mathcal{O}\left(\delta_{h}^{2}\right)$. Subject to

$$
\begin{aligned}
0 \geq \mathbf{c}^{+}\left(\mathbf{z}^{*+}\right)= & \left(G+\partial_{h} G \delta_{h}\right) \mathbf{z}^{*+}- \\
& W-\left(Y+\partial_{h} Y \delta_{h}\right) x_{i}+\mathcal{O}\left(\delta_{h}^{2}\right)
\end{aligned}
$$

with a value function $J_{z}^{+}=\ell_{z}^{+}\left(\mathbf{z}^{*+}\right)$. The perturbed solution is $\mathbf{z}^{*+}=-\left(H+\partial_{h} H \delta_{h}\right)^{-1}\left(G+\partial_{h} G \delta_{h}\right)^{\top} \boldsymbol{\mu}^{+}+\mathcal{O}\left(\delta_{h}^{2}\right)$. Substituting (A.1) yields a solution of the form $\mathbf{z}^{*+}=$ $\mathbf{z}^{*}+\partial_{h} z \delta_{h}+\mathcal{O}\left(\delta_{h}^{2}\right)$. A similar sequence of derivations can be followed for $T, T^{+}=T+\delta_{T}$, affecting $\bar{Q}_{\mathrm{r}}, \bar{R}_{\mathrm{r}}$ and $\bar{S}_{\mathrm{r}}$ and yielding the perturbed system of the form (B.1). The solution can consequently be expressed as

$$
\mathbf{z}^{*+}=\mathbf{z}^{*}+\partial_{h} \mathbf{z}^{*} \delta_{h}+\partial_{T} z \delta_{T}+\mathcal{O}\left(\delta_{h}^{2}, \delta_{T}^{2}\right)
$$

for some finite $\partial_{h} z$ and $\partial_{T} z$. Hence, $\mathbf{z}^{*}$ is differentiable.

Case 2 (Changing $N$ ) Now consider a perturbation $h^{+}:=h+\delta_{h}$ as $0<\delta \rightarrow 0$ applied to an OCP with $N-1$ prediction steps and $h_{\mathrm{r}}=h$. The unperturbed OCP has the following matrices related to (8) and (9): $\widetilde{\mathbf{B}}=\left[\underline{\widetilde{B}} \widetilde{B}_{\bullet}\right]^{\top}, \quad \widetilde{\mathbf{Q}}=\operatorname{diag}\left(\underline{\widetilde{Q}}, Q_{f}\right), \quad \mathbf{R}=\underline{R}, \mathcal{R}=\underline{\mathcal{R}}$. Now consider a perturbation $h^{-}=h-\delta_{h}$ as $0<\delta \rightarrow$ 0 on an OCP with $N$ prediction steps and $h_{\mathrm{r}}=0$. The unperturbed OCP now has $\widetilde{\mathbf{B}}=\left[\begin{array}{ccc}\widetilde{B} & \widetilde{B} & \widetilde{B} \\ 0 & 0 & 0\end{array}\right]^{\top}, \widetilde{\mathbf{Q}}=$ $\operatorname{diag}\left(\underline{\widetilde{Q}}, 0, Q_{f}\right), \mathbf{R}=\left[\underline{R}_{0}\right], \mathcal{R}=\left[\underline{\mathcal{R}}_{0}\right]$. After some algebra, it can be shown that the solution $\mathbf{z}^{*}=-H^{-1} G^{\top} \boldsymbol{\mu}$ associated with these block matrices are

$$
\begin{aligned}
& \mathbf{z}_{(1)}^{*}=\alpha\left[(I-\mathcal{R} \underline{\widetilde{B}})-(I-\mathcal{R} \underline{\widetilde{B}}) \underline{\widetilde{B}} \overrightarrow{\widetilde{B}}-\underline{\widetilde{B}}-\widetilde{\widetilde{B}}_{\bullet}\right] \boldsymbol{\mu}, \\
& \mathbf{z}_{(2)}^{*}=\left[\begin{array}{c}
\alpha\left[(I-\mathcal{R} \underline{\widetilde{B}}) 0-(I-\mathcal{R} \underline{\widetilde{B}}) 0 \underline{\widetilde{B}} \widetilde{B}_{\bullet} \widetilde{B}_{\bullet}-\underline{\widetilde{B}}-\widetilde{B}_{\bullet}-\widetilde{B}_{\bullet}\right] \\
0
\end{array}\right] \boldsymbol{\mu}
\end{aligned}
$$

respectively, where $\alpha:=-2\left(\underline{\widetilde{B}}^{\top} \underline{\widetilde{Q}} \underline{\widetilde{B}}+\widetilde{B}_{\bullet}^{\top} Q_{f} \widetilde{\widetilde{B}} \cdot+\underline{R}\right)$. The two equations in (B.3) yields the same solution (the residual step in $\mathbf{z}_{(2)}^{*}$ contributes only addition of zeroes). However, the rate of change over the perturbation is not.

For the OCP with $N$ prediction steps and $h_{\mathrm{r}}=0$, the rate of change of $\mathbf{z}^{*}$ w.r.t. $h$ can be obtained from $\partial_{h} \mathbf{z}_{(2)}^{*}$ before the substitution $h_{\mathrm{r}}=0$. This would have additional terms compared to $\partial_{h} \mathbf{z}_{(1)}^{*}$ of that with $N-1$ steps. These terms are associated with the constraints for the additional prediction step and will be zero only if the additional constraints are inactive so that the Lagrange multipliers and their rates of change are zero. Hence, the solution cannot be expressed as (B.2) in general, since $\partial_{h} \mathbf{z}^{*}$ is not guaranteed to be unique and differentiability of $\mathbf{z}^{*}$ at changing $N$ is not guaranteed. A similar result is obtained when looking at the rate of change w.r.t. $T$. 